\title{
Thermal Boundary Conductance between Multi-Walled Carbon Nanotubes*
}

\author{
Yutaka YAMADA**, Takashi NISHIYAMA**, Takahiro YASUHARA** \\ and Koji TAKAHASHI** \\ **Department of Aeronautics and Astronautics, Faculty of Engineering, Kyushu University, \\ 744 Motooka, nishi-ku, Fukuoka, 819-0395, Japan \\ E-mail: nishiyama@aero.kyushu-u.ac.jp
}

\begin{abstract}
Interfacial thermal transport of multi-walled carbon nanotubes (MWNTs) is investigated by using bulk pellet specimens. Steady-state conduction method gives thermal conductivity of 1 to $4 \mathrm{~W} / \mathrm{mK}$ for the pellets with mass density from 0.2 to $0.35 \mathrm{~g} / \mathrm{cm}^{3}$. This low thermal conductivity is due to the thermal boundary conductance between the nanotubes. Computational analysis is conducted for the pellet modeled as a random network of spherocylinders (SCs) and calculated dependency of thermal conductivity on pellet density shows good agreement with experimental data when we treat non-uniform SCs. By comparing the experimental and computational results, the thermal boundary conductance between two MWNTs can be taken as $1.5 \times 10^{-8} \mathrm{~W} / \mathrm{K}$. This result agrees well with the reported data obtained by individual measurement, which suggests this simple method is applicable to probe the interfacial thermal phenomena of nanomaterials. An improved scaling law, $\mathrm{k} \propto \rho^{2.14}$, for thermal conductivity of MWNTs aggregations is also proposed and discussed.
\end{abstract}

Key words: Carbon Nanotubes, Thermal Boundary Conductance, Thermal Conductivity

\section{Introduction}

The distinguished properties of carbon nanotube (CNT) have been attracting many researchers of a variety of fields since its discovery ${ }^{(1)}$. For thermal engineering, its great heat conduction performance in its axial direction is extremely promising. So far, experimental studies have reported several thousand $\mathrm{W} / \mathrm{mK}$ for both single-walled carbon nanotubes (SWNTs) ${ }^{(2,3)}$ and thin multi-walled carbon nanotubes (MWNTs) ${ }^{(4,5)}$, which has been confirmed by recent theoretical studies ${ }^{(6)}$. For thick MWNTs, thermal conductivity is reduced with increase in diameter, but they are still higher than $100 \mathrm{~W} / \mathrm{mK}^{(5,7)}$, which makes them useful as filler in nanocomposites or nanofluids to improve thermal transport.

Thermal interface materials (TIMs) ${ }^{(8,9)}$ is another promising application of CNT to transfer heat between heat spreader (heat sink) and heat source (CPU or other electronic devices). The thermal conductivity of TIM using vertically aligned (VA) CNT arrays was reported $74 \sim 83 \mathrm{~W} / \mathrm{mK}$ in the temperature range of $295 \sim 323 \mathrm{~K}$ by an experimental study ${ }^{(10)}$, which is an order of magnitude higher than thermal grease. However, the bulk CNT rarely shows thermal conductivity higher than $100 \mathrm{~W} / \mathrm{mK}$ even using vertically aligned (VA) CNT arrays. The experimentally obtained data for buckypaper and CNT pellets are in the range of $0.1 \sim$ several $\mathrm{W} / \mathrm{mK}$. Unfortunately, the mechanism for heat conduction of these bulk materials is not clear. Even for the three-dimensional random network of CNTs, there are few reports ${ }^{(11-13)}$. A quadratic law of thermal conductivity on mass density was proposed by

${ }^{*}$ Received 8 Dec., 2011 (No. 11-0761) [DOI: 10.1299/jtst.7.190]

Copyright $@ 2012$ by JSME 
Volkov and Zhigilei ${ }^{(12)}$ using a simple model. However more reliable prediction of thermal conductivity of bulk CNT material is desired.

Basically, contact issue is vital for microscale heat transfer in these applications and has been treated by molecular dynamics simulation ${ }^{(14)}$, but the result strongly depends on the assumption represented by the nanotube-nanotube distance, which means that our knowledge on interface thermal transport is very limited. Recently, individual contact thermal conductance of MWNTs was successfully measured by using a MEMS-based sensing device ${ }^{(7)}$. But it is extremely difficult for general readers to apply their technique. In this paper, we simultaneously conduct experimental and computational studies on an identical pellet specimen of MWNTs aggregation and propose a simple method to estimate the thermal boundary conductance between fibrous nanomaterials represented by CNT.

\section{Measurement of bulk thermal conductivity}

MWNT powder synthesized by CVD method is pressed to form the pellet specimen. The length and diameter of the MWNTs is determined by using a high-resolution transmission electron microscope (HRTEM, JEM-3200FSK) and their average values are approximately (length) $10 \mu \mathrm{m}$ and (diameter) $100 \mathrm{~nm}$. After measuring the mass of the pellet using an electronic balance, it is sandwiched between two brass rods and its density is controlled by the applied load. The rod ends are coated with thin silicone grease films of high thermal conductivity, whose thickness is less than $10 \mu \mathrm{m}$. The load is kept constant during a measurement and the specimen thickness is determined by an in-situ measurement system utilizing a combination of a digital camera and microscope lenses. The thickness of the pellet is measured to an accuracy of $10 \mu \mathrm{m}$ by comparison with a microscale previously recorded at the same magnification. Thickness range of the pellets from 400 to $1000 \mu \mathrm{m}$ and specimen mass density from 0.20 to $0.35 \mathrm{~g} / \mathrm{cm}^{3}$ is measured.
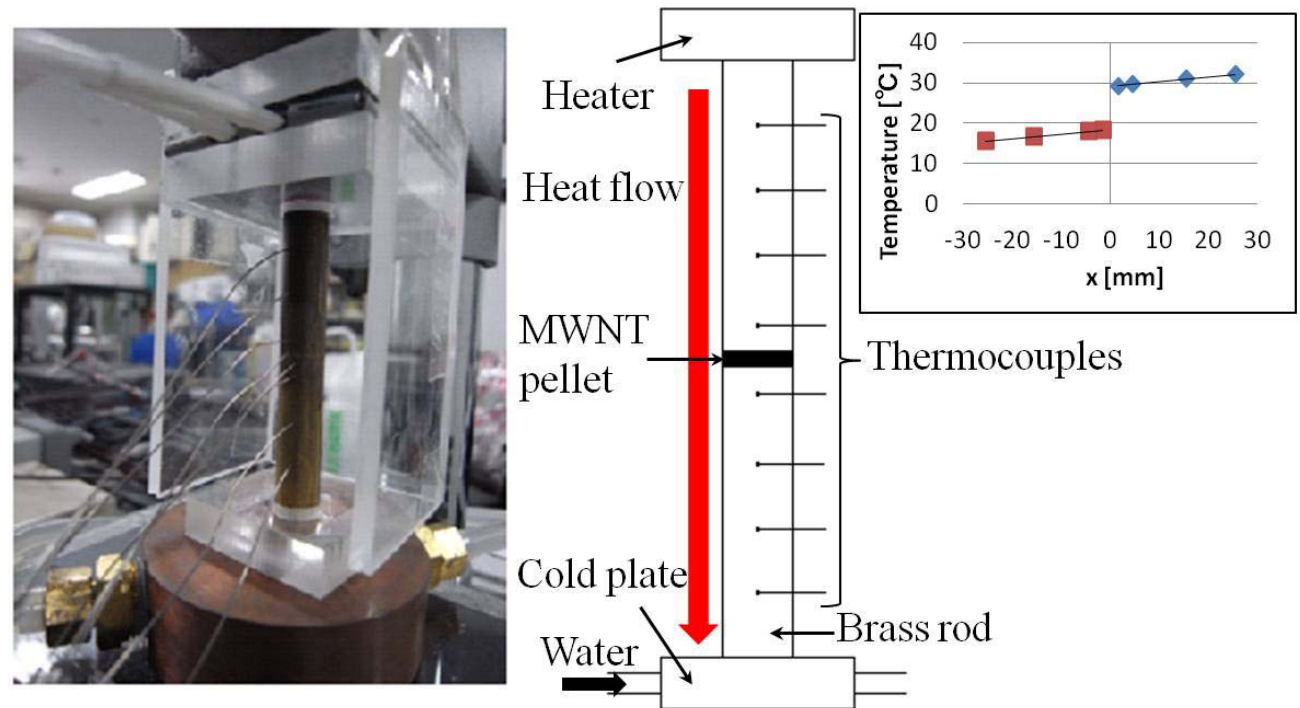

Fig.1 Experimental setup of thermal conductivity measurement. The thermal conductivity of brass rods is $117 \mathrm{~W} / \mathrm{mK}$. Inset shows the temperature distribution of rod.

Our experimental setup is shown in Fig. 1. The brass rods (11 $\mathrm{mm}$ in diameter) are employed as the reference material for thermal conductivity measurement. The ends of the rods are mirror polished surfaces. There are small holes for temperature measurement $(0.6$ $\mathrm{mm}$ diameter and $5 \mathrm{~mm}$ depth) on the side of the brass rods, and the holes are located at distances of $1 \mathrm{~mm}, 4 \mathrm{~mm}, 15 \mathrm{~mm}, 25 \mathrm{~mm}$, and $35 \mathrm{~mm}$ from the edge of the specimen side 
of the brass rod, respectively. Where a pellet is sandwiched with rods, the upper end of the upper rod is heated with a ceramic heater, and the lower end is cooled with a copper plate that is kept at a constant temperature by circulation of cold water. The load applied to the pellet is mechanically adjusted by up-and-down movement of the lower cool stage. $T$ type sheathed thermocouples were used for temperature measurement. The thermocouples with a sheath diameter of $0.5 \mathrm{~mm}$ are set into the holes on the side of the rod. Heat dissipation through them is negligible. The temperature of each point of measurement is displayed on a data logger. The temperatures are recorded after waiting for the temperature to stabilize (about $20 \mathrm{~min}$.).

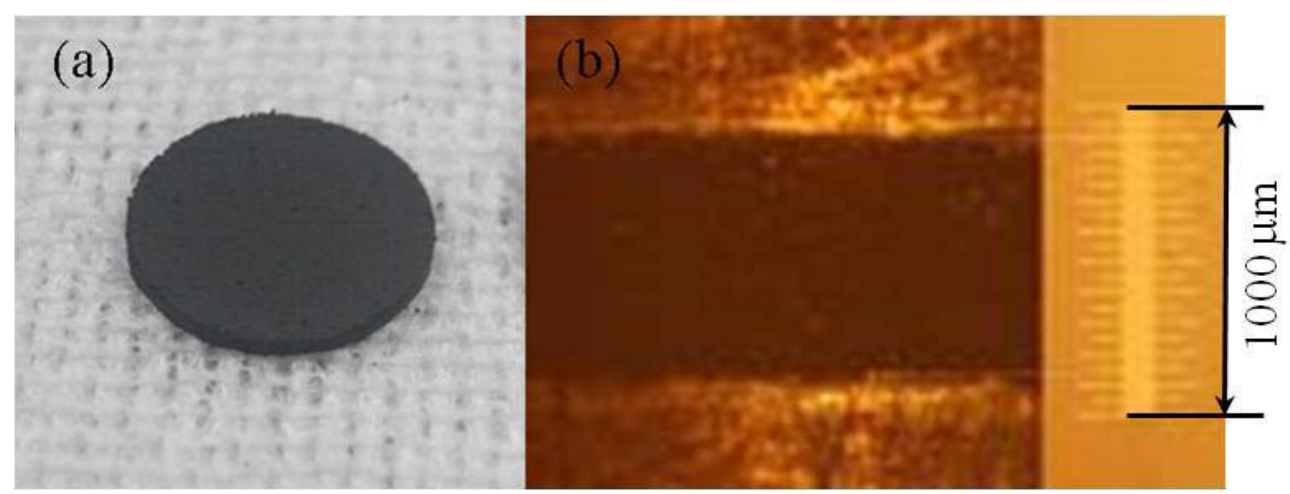

Fig.2 CNT pellet (a) and a zoomed picture of the pellet thickness measurement, where the thickness is 780 $\mu \mathrm{m}$, (b).

Heat flows through the brass-pellet-brass under room temperature. In order to suppress convection, the surroundings of the brass rod and pellet specimen are covered with a thermal insulator made of polyethylene foam, whose thickness is $10 \mathrm{~mm}$. Consequently one-dimensional steady-state heat conduction can be assumed there. The temperature gap of the specimen was obtained by extrapolating the temperature distribution of the brass rod. Using the known thermal conductivity of the brass rod, heat flux was calculated and the thermal conductivity of the pellet specimen was estimated. It was confirmed by using reference material that we can measure thermal conductivity in triple digits accuracy. As seen in Fig. 2, the measured specimen is confirmed to be uniform without any cracks and voids. The mass density of the pellet is estimated from the mass of the used MWNTs and the volume of the pellet. The MWNTs mass is measured by an electronic balance before forming the pellet which is calculated from its thickness determined by the in-situ measurement system.

The measured thermal conductivity was $1 \sim 4 \mathrm{~W} / \mathrm{mK}$. This value is several orders lower than that of individual CNTs due to the low thermal boundary conductance. In other word, the present heat conduction of non-orientated CNT pellets is independent on the intrinsic thermal conductivity of CNTs, but is dominated by the thermal boundary conductance between CNTs. This scenario is used in the next section for computational modeling.

\section{Computational approach for thermal boundary conductance}

We treat spherocylinders (SCs) for MWNTs and a three-dimensional random network of SCs for the pellet. Figure 3 shows the configuration of the SCs and an example of a calculated temperature distribution. The shape of the $\mathrm{SC}$ is a rod with a spherical hemisphere on both ends of the cylinder. In this simulation, $i$-th $\mathrm{SC}$ is designated $\mathrm{SC}_{i}, r_{i}$ is the radius of $\mathrm{SC}_{i}$, and $l_{i}$ is the length of the pillar part of the SC. The size of the calculation area is $10000 \times 10000 \times 50000 \mathrm{~nm}$. MWNT pellet is simulated by $N$ pieces of site (center of 
the SC) that are placed by using uniform random numbers in the calculation area. The $N$ value is set from 5000 to 11000 in order to control the density. In Fig.3 (b), 10000 SCs are arranged on the sites. The orientation angles of the $\operatorname{SCs}\left(\theta_{i}, \phi_{i}\right)$ are determined by using uniform random numbers, because the pellets used in the experimental measurement are made from non-oriented MWNTs. Its randomness is confirmed by SEM image as listed in Fig.3(c) and (d). After determining the form of the SCs, calculation area, arrangement of site, and orientation angle, the geometric arrangement of all the SCs is investigated and heat conduction routes are decided. In this simulation, the existence of a heat conduction route is taken to be the existence of contact between the $i$-th and $j$-th SC.

(a)

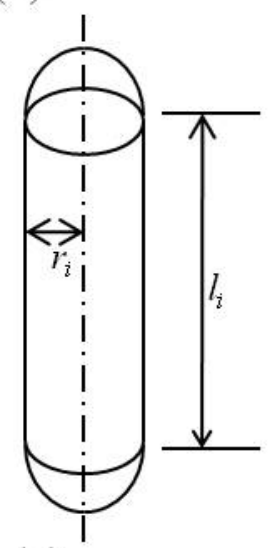

(c)

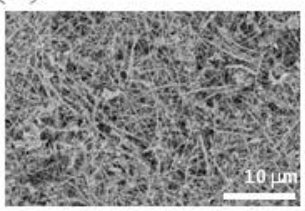

(b)

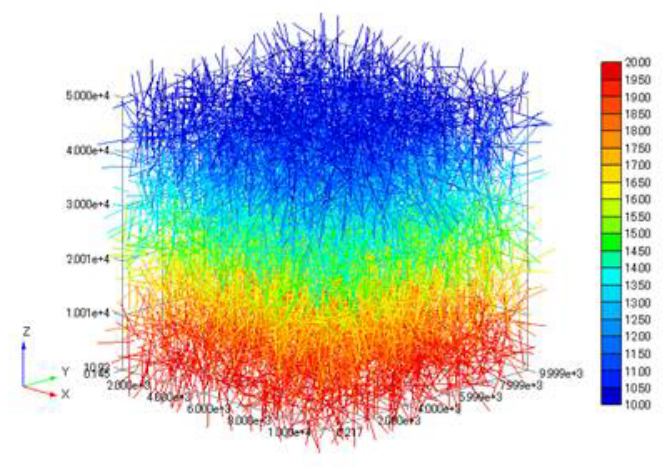

(d)

(e)

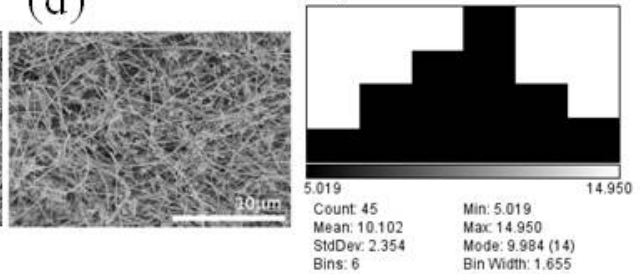

Fig.3 (a) Schematic of a spherocylinder, which consists of a cylinder of length $l_{i}$ and two hemispheres of radius $r_{i}$. (b) Calculated temperature distribution using 10000 SCs. (c) SEM image of $x y$-plane of measured pellet. (d) $x z$-plane. (e) Distribution of CNT length.

Additionally, in order to calculate density for the simulated pellet, the mass of all the $\mathrm{SCs}$ in the area is required. So, the mass of $\mathrm{SC}_{\mathrm{i}}$ and the mass of the whole pellet are estimated from the atomic weight of carbon and the number of layers of graphene that forms the MWNT.

As seen there, each SC is assumed to have an identical temperature throughout. CNT has a large thermal conductivity in the direction of the long axis. So the temperature in each CNT is set constant. The heat flow of the network system is dependent only on the thermal boundary conductance at SCs junctions. Therefore inter-tube heat flow $Q_{i j}$ is given by Fourier's law,

$$
Q_{i j}=\sigma_{c, i j}\left(T_{j}-T_{i}\right)
$$

where $Q_{i j}$ is the heat flow between the $i$-th and $j$-th CNT. $T_{i}$ and $T_{j}$ are temperatures of each of the contacted SCs and $\sigma_{c, i j}$ is the thermal boundary conductance. In this model, we assume constant thermal boundary conductance for all junctions; $\sigma_{c i j}=\sigma_{c}$. The thermal conductivity $k_{S C}$ of the network system is defined as, 


$$
k_{S C}=-\frac{q_{z}}{d T / d z}
$$

where $q_{z}$ is the z-axis heat flux, defined by $q_{z}=Q_{z} / L_{x} L_{y} . Q_{z}$ is the average heat flow through the computational region along the z-axis with $L_{x} L_{y}$ as its cross section.

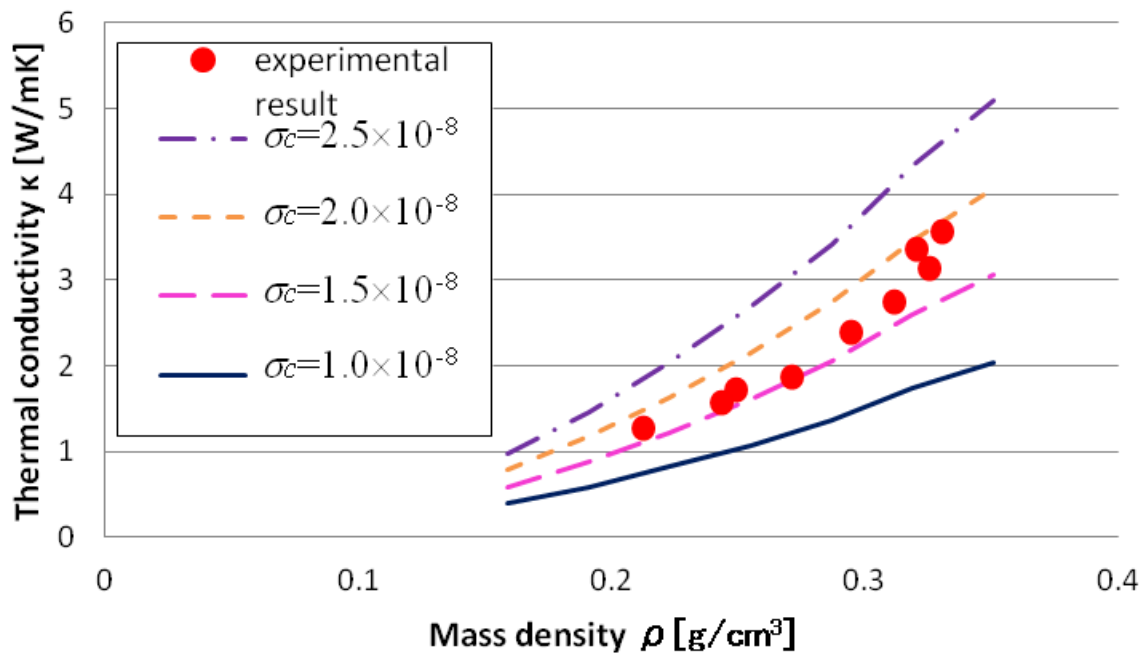

Fig.4 Calculated thermal conductivity of random network homogeneous SCs in comparison with the experimental results. Thermal boundary conductance of $1.0,1.5,2.0,2.5 \times 10^{-8} \mathrm{~W} / \mathrm{K}$ was used.

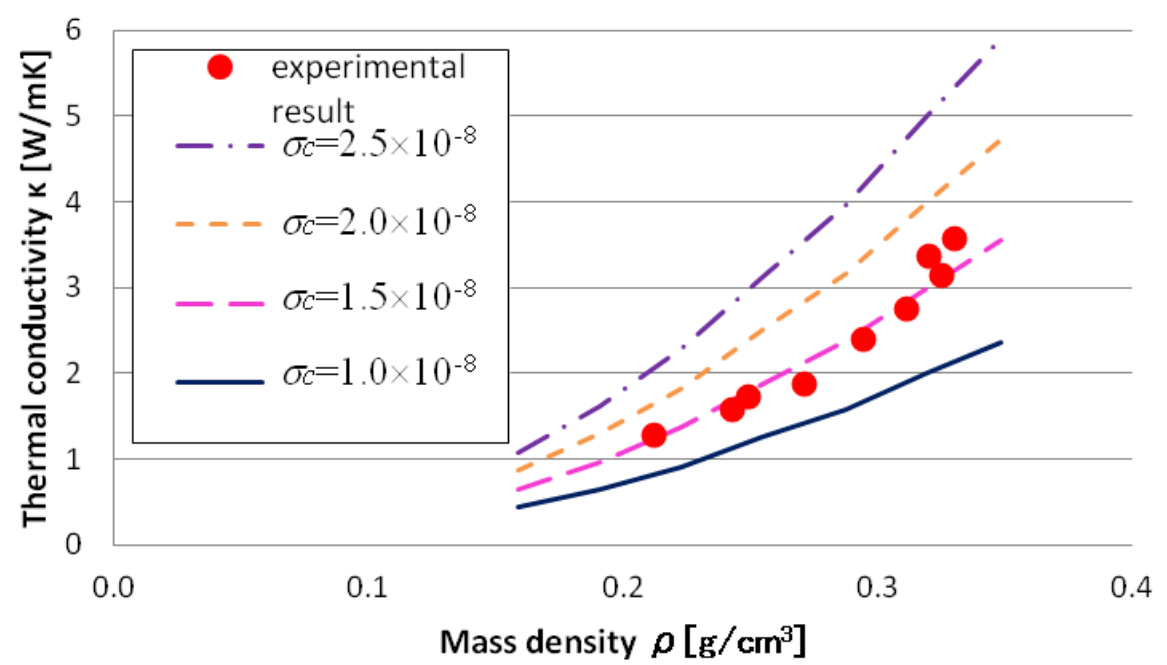

Fig.5 Calculated thermal conductivity of inhomogeneous random network SCs. Thermal boundary conductance of $1.5 \times 10^{-8} \mathrm{~W} / \mathrm{K}$ shows the best fit with experimental data.

The size of SCs is determined by their images obtained by HRTEM. For an aligned contact of MWNTs, the thermal boundary conductance was estimated to be on the order of $10^{-6} \mathrm{~W} / \mathrm{K}$, while that for a cross contact of MWNTs with smaller contact area was on the order of $10^{-8} \mathrm{~W} / \mathrm{K}^{(7)}$. As will be discussed later, most CNTs in our pellets show cross contacts thus we used $1.0 \sim 2.5 \times 10^{-8} \mathrm{~W} / \mathrm{K}$ in our calculation. In order to obtain a minimum 
approach distance $d_{\min }$ between two vectors of SC, a fast algorithm to evaluate the shortest distance between rods was used ${ }^{(15)}$. When $d_{\min }$ is smaller than the summation of two SCs radii, two rods are assumed to contact.

Calculated thermal conductivity of SCs of identical lengths of $10 \mu \mathrm{m}$ and radius of 50 $\mathrm{nm}$ is listed in Fig. 4 together with the experimental data. Simulation results are in the same range as the experimental result, which suggests that the reported thermal boundary conductance $^{(7)}$ is reliable. It is also found that the curve of computational result does not fit the experimental data, which problem is probably due to the simple modeling. Next we developed an improved model treating the distribution of length and radius according to the HRTEM and SEM observation, which show that the actual MWNTs are not homogenous. The length of randomly chosen 45 CNTs are carefully measured from SEM image and actual distribution is listed in Fig. 3(e). The mean value of length is found about $10 \mu \mathrm{m}$. The radius of 10 CNTs are measured using TEM. Its mean value is about $50 \mathrm{~nm}$. Because the CNTs are not straight, error is always exists in the measurement. We assumed the length and radius ranges as normal distribution of $5 \sim 15 \mu \mathrm{m}$ and $35 \sim 65 \mathrm{~nm}$ respectively. Calculated results are listed in Fig. 5, which show a better fit to the experimental data. From this comparative study, we can conclude that the thermal boundary conductance in our pellets is approximately $1.5 \times 10^{-8} \mathrm{~W} / \mathrm{K}$. Though the fitting is dependent on the distributed profile of length and radius of CNTs, we determined it only from one samples. The reliability of this data has to be discussed after measuring more samples.

\section{Discussion}

So far, reliable data of thermal boundary conductance of CNTs are very limited. One is that reported by Yang et al. ${ }^{(7)}$, where thermal boundary conductance is reported on the order of $10^{-8} \mathrm{~W} / \mathrm{K}$ between two individual MWNT of cross contact. Another measurement was conducted by Hirotani et al. ${ }^{(16)}$, where thermal boundary conductance between an individual MWNT end and Au surface was found ca. $10^{-7} \mathrm{~W} / \mathrm{K}$. Our result agrees well with the former one and also the latter one is consistent with our data when considering the conductance per unit area because the contact area of the MWNT end is about one-order smaller than the present side-wall contact.

For the reliability of thermal boundary conductance estimated from the present method, modeling of the heat conduction of a random network of CNTs is important. Both our experimental and computational results shows good agreement with the quadratic relationship derived for a non-oriented 3D system in Ref. (12). We further looked into the scaling law that best fit our computational results as shown in Fig. 6, where we used a non-dimensional density parameter $\bar{n}=n L^{2} R$ according to the theoretical model in Ref. (12). $n$ is the volume number density, $L$ is the length, and $R$ is the external radius of the SCs. For the calculation of inhomogeneous SCs, $L$ and $R$ are the averaged values. 


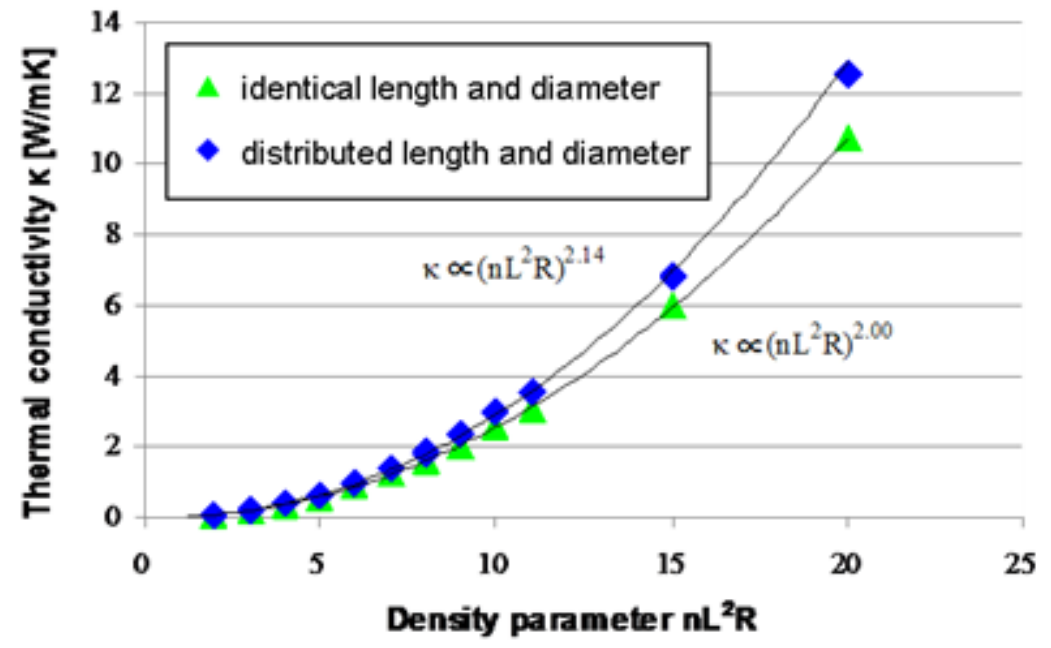

Fig.6 Scaling law for numerically obtained thermal conductivity. Green triangles are for SCs of uniform size and blue for ones of non-uniform size.

For the network of homogeneous fibers, the profile of thermal conductivity shows good agreement with a scaling law of the form $k \propto \bar{n}^{2.00}$, which is consistent with the quadratic law ${ }^{(12)}$. However the profile for the inhomogeneous fibers deviates slightly from this relationship and is estimated as $k \propto \bar{n}^{2.14}$. Because our experimental data shows much better fit with the inhomogeneous calculation, we propose here that the power-law index derived in Ref. (12) has to be modified from 2.00 to 2.14 .

Here we discuss a physical interpretation of 2.14. At the distributed SCs, average of $L^{2}$ in the non-dimensional density parameter $n L^{2} R$ is expected to become larger than that of identical SCs and a power-law index should increase. And physically, longer and wider SCs contribute more effectively than shorter and thinner ones in the current distributed thermal resistances.

In order to improve the reliability of thermal boundary conductance data, we have to reconsider our computational modeling. Our SCs model assumed the same contact area as an identical thermal boundary conductance. But it is obvious that a non-negligible fraction of CNT contacts should be of larger area than the cross-contact because the real CNTs are flexible and van der Waals force works as attraction force. Figure 7 is an SEM image of the measured pellet, where many bundled or agglutinated CNTs are included and more critically CNTs tend to form aggregations. The flexibility of CNT should increase the thermal conductivity but the large gaps created between the aggregated CNTs yield large thermal resistance. These unexpected issues can cause a large divergence in estimating thermal boundary conductance of nanomaterials. In addition, tightly bundled CNTs are seen but their volume is less than $3 \%$ of the whole pellet, whose effect is smaller than the errors caused by thickness measurement etc. Therefore we assume that CNTs are distributed randomly in this simulation. Figure 7 indicates the existence of voids in the pellet too. However, thermal conductivity of CNT is four or more order of magnitude larger than that of air. Thus the present work neglects the effect of void but further investigation is required together with an improved analysis. 


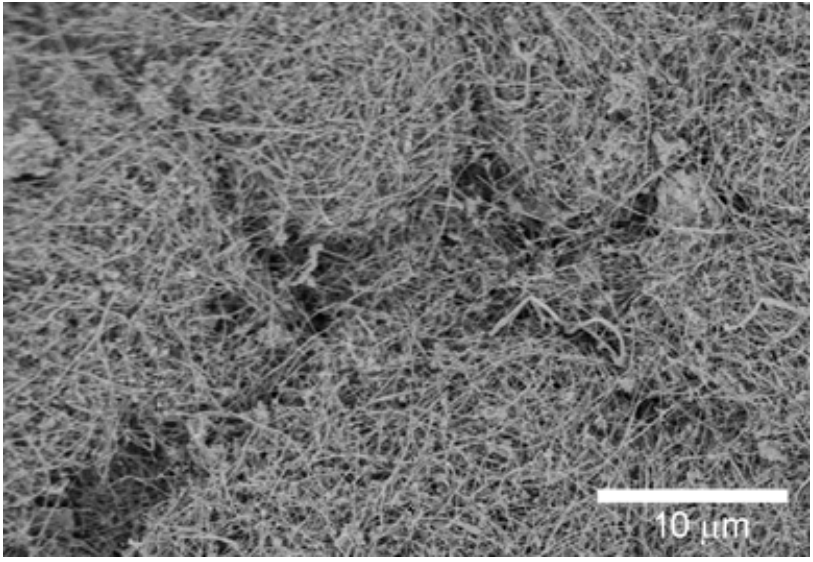

Fig.7 SEM image of measured CNT pellet, where bundled nanotubes and aggregated CNTs with large gaps are seen.

\section{Conclusion}

In order to obtain the thermal boundary conductance of MWNTs, we conducted both experimental and computational approaches on thermal conductivity of pellet. By using brass rods assuming one-dimensional heat conduction, thermal conductivity values of 1 4 $\mathrm{W} / \mathrm{mK}$ were obtained for a density range of $0.2 \sim 0.35 \mathrm{~g} / \mathrm{cm}^{3}$. Computational analysis treats SCs of reliable size data of MWNTs from HRTEM observation by assuming an identical thermal boundary conductance between CNTs. Results using inhomogeneous random network SCs match with experimental data. From their comparison, the thermal boundary conductance is estimated as $1.5 \times 10^{-8} \mathrm{~W} / \mathrm{K}$, which agrees well with previously reported experimental data using individual MWNTs. A new scaling law in the form $k \propto \bar{n}^{2.14}$ has been derived from the data. The deviation from the quadratic law is found to come from the non-uniformity of CNTs. SEM image of the pellets suggests that further improvement is required to predict the thermal boundary conductance more precisely by considering CNT bundles and gaps.

\section{Acknowledgements}

The authors acknowledge the financial support from the Grants-in-Aid for Scientific Research $(23360101,23656153,23760191)$. The HRTEM observations were conducted in the Research Laboratory for High Voltage Electron Microscopy, Kyushu University. The help of Mr. Tatsuya Ikuta for HRTEM and SEM experiments is also gratefully acknowledged.

\section{References}

(1) Iijima, S. (1991) Helical microtubules of graphitic carbon, Nature, 354.56

(2) Yu, C., Shi, L., Yao, Z., Li, D. and Majumdar, A., (2005) Thermal Conductance and Thermopower of an Individual Single-Wall Carbon Nanotube, Nano Letters, 5. 9. 1842

(3) Pop, E., Mann, D., Wang, Q., Goodson, K. and Dai, H., (2006) Thermal Conductance of an Individual Single-Wall Carbon Nanotube above Room Temperature, Nano Letters, 6. 1.96

(4) Kim, P., Shi, L., Majumdar, A. and McEuen, P. L., (2001) Thermal Transport Measurements of Individual Multewalled Nanotubes, Physical Review Letters, 87. 215502

(5) Fujii, M., Zhang, X., Xie, H., Ago, H., Takahashi, K., Ikuta, T., Abe, H. and Shimizu, T., (2005) Measuring the Thermal Conductivity of a Single Carbon Nanotube, Physical Review Letters, 95. 065502 
(6) Mingo, N. and Broido, D. A., (2005) Length Dependence of Carbon Nanotube Thermal Conductivity and the "Problem of Long Waves", Nano Letters, 5. 7. 1221

(7) Yang, J., Waltermire, S., Chen, Y., Zinn, A. A., Xu, T. T. and Li, D., (2010) Contact thermal resistance between individual multiwall carbon nanotubes, Applied Physics Letters, 96. 023109

(8) Tong, T., Zhao, Y., Delzeit, L., Kashani, A., Meyyappan, M. and Majumder, A., (2007) Dense Vertically Aligned Multiwalled Carbon Nanotube Arrays as Thermal Interface Materials, IEEE Transaction on components and packaging technologies, 30. 1.92

(9) Panzer, M. A., Zhang, G., Mann, D., Hu, X., Pop, E., Dai, H. and Goodson, K. E., (2008) Thermal Properties of Metal-Coated Vertically Aligned Single-Wall Nanotube Arrays, Journal of Heat Transfer, 130. 052401

(10) Hu, X. J., Padilla, A. A., Xu, J., Fisher, T. S. and Goodson, K. E., (2006) 3-Omega Measurements of Vertically Oriented Carbon Nanotubes on Silicon, Journal of Heat Transfer, 128. 1109

(11) Prasher, R. S, Hu, X. J., Chalopin, Y., Mingo, N., Lofgreen, K., Volz, S., Cleri, F. and Keblinski, P., (2009) Turning Carbon Nanotubes from Exceptional Heat Conductors into Insulators, Physical Review Letters, 102, 105901

(12) Volkov, A. N. and Zhigilei, L. V., (2010) Scaling Laws and Mesoscopic Modeling of Thermal Conductivity in Carbon Nanotube Materials, Physical Review Letters, 104. 215902

(13) Chalopin, Y., Volz, S. and Mingo, N., (2010) Erratum: "Upper bound to the thermal conductivity of carbon nanotube pellets" [J. Appl. Phys. 105, 084301 (2009)], Journal of Applied Physics, 108, 039902

(14) Zhong, H. and Lukes, J. R., (2006) Interfacial thermal resistance between carbon nanotubes: Molecular dynamics and analytical thermal modeling, Physical Review B, 74. 125403

(15) Vega, C. and Lago, S., (1994) A fast algorithm to evaluate the shortest distance between rods, Computers \& Chemistry, 18. 1. 55

(16) Hirotani, J., Ikuta, T., Nishiyama, T. and Takahashi, K., (2011) Thermal boundary resistance between the end of an individual carbon nanotube and a Au surface, Nanotechnology, 22 (31), art. no. 315702 